\title{
Efficacy of the Treatment of Intraperitoneal Bladder Perforation during Transurethral Resection of Bladder Tumor with the Urethral Catheter Alone: Retrospective Analysis of over 15 Years Using the Clinical Data Warehouse System
}

\author{
Junghoon Lee ${ }^{a}$ Jungyo Suh ${ }^{b}$ Chang Wook Jeong ${ }^{b}$ Cheol Kwak ${ }^{b}$ \\ Hyeon Hoe Kim ${ }^{\mathrm{b}}$ Ja Hyeon $\mathrm{Ku}^{\mathrm{b}}$ \\ ${ }^{a}$ Department of Urology, Kangdong Sacred Heart Hospital, Seoul, South Korea; ${ }^{b}$ Department of Urology, Seoul \\ National University Hospital, Seoul, South Korea
}

\section{Keywords}

Bladder perforation - Neoplasm · Urothelial carcinoma .

Transurethral resection . Clinical Data Warehouse

\begin{abstract}
Introduction: We investigated the efficacy of a urethral catheter alone for intraperitoneal perforation during transurethral resection of bladder tumor (TURBT). Patients and Methods: We retrospectively evaluated the medical records of 4,543 patients who underwent TURBT from January 2000 to December 2017 using the Clinical Data Warehouse system. The clinicopathologic characteristics, recurrence-free survival, and progression-free survival were compared between the patient groups with intraperitoneal perforation treated with the Foley catheter alone, extraperitoneal perforation, and matched control TURBT. Results: Intraperitoneal perforation and extraperitoneal perforation were observed in $16(35.6 \%)$ and $29(64.4 \%)$ patients, respectively. In the intraperitoneal perforation group, 11 (68.8\%), 2 (12.5\%), and 3 (18.8\%) patients were treated with the Foley catheter alone, additional percutaneous drainage, and delayed open sur-
\end{abstract}

gery, respectively. The use of the Foley catheter alone in patients with intraperitoneal perforation of smaller size than the cystoscope or no pelvic radiotherapy history showed improved efficacy without sequelae or therapeutic delay. One of the 2 patients with the size of the intraperitoneal perforation larger than the cystoscope was successfully treated with the Foley catheter alone, whereas the other patient underwent delayed surgical repair. There was no difference in recurrence-free survival and progression-free survival of the intraperitoneal perforation treated with the Foley catheter alone compared to those of the matched control TURBT ( $p=$ $0.909, p=0.518)$ and the extraperitoneal perforation ( $p=$ $0.458, p=0.699)$. Conclusions: Intraperitoneal perforation rarely occurred during TURBT. In the case of intraperitoneal perforation of size smaller than cystoscopy or without pelvic radiotherapy history, treatment with the Foley alone showed successful improvement and safe oncological results. Therefore, treatment with the urethral catheter alone can be carefully considered when an intraperitoneal perforation smaller than the cystoscope size or without pelvic radiotherapy history occurs.

(C) 2021 The Author(s)

Published by S. Karger AG, Basel
(C) 2021 The Author(s)

Published by S. Karger AG, Basel

This is an Open Access article licensed under the Creative Commons Attribution-NonCommercial-4.0 International License (CC BY-NC) (http://www.karger.com/Services/OpenAccessLicense), applicable to the online version of the article only. Usage and distribution for commercial purposes requires written permission.
Correspondence to:

Ja Hyeon Ku, kuuro70@snu.ac.kr 


\section{Introduction}

Transurethral resection of bladder tumor (TURBT) is the most common procedure for the diagnosis and treatment of bladder cancer because it permits minimally invasive lesion excision and allows patients to quickly regain their daily activities. Although TURBT complications are rare, there exist chances of the occurrence of severe bladder perforation or tumor cell implantation [1, 2]. Postoperative fluid irrigation, intravesical treatment, catheter drainage, and surgical repair are the existing techniques to prevent these complications and tumor recurrence $[3,4]$. Perforation during TURBT occurs in approximately $0.9-5 \%$ of cases, and $85 \%$ of perforations are classified as extraperitoneal perforation (EPP). Generally, EPP is expected to improve with conservative management [5]. However, rarely occurring intraperitoneal perforations (IPPs) require surgical repair owing to their potential for complications [6].

In case of the occurrence of IPP, the appropriate treatment method is chosen based on the risk of fluid leakage, bleeding, and tumor cell extravesical implantation. Recently, minimally invasive treatment is gaining grounds as the treatment of choice, as surgical repair is a risk factor for tumor progression [4]. Consequently, treatment options, such as percutaneous drainage (PCD) and Foley catheter drainage, are commonly employed [7]. However, owing to the low perforation risk and the retrospective nature of studies conducted on this topic, the clinical course and prognosis of the patients remain unclear, because long-term clinical cohort studies with low incidence are difficult to conduct under clinical settings.

A data warehouse, defined as "a subject-oriented, integrated, nonvolatile, and time-variant collection of data in support of management decisions," was first developed in the 1980s [8]. In the electronic health record (EHR), a great extent of valuable data are fragmented into small pieces such that information acquisition becomes difficult. Extracting EHR data from a particular case could be helpful in disease management. The Clinical Data Warehouse (CDW) is an information technology tool that integrates and utilizes data from clinical operation systems [9-11]. It can detect and stratify disease risk and prevent and monitor disease progression for disease management. Typically, CDW extracts, cleanses, and summarizes EHR data, which is beneficial to the researchers.

Compared to the current conservative treatment method in case of EPP occurrence during TURBT, the treatment method of IPP is more aggressive. Therefore, it is necessary to compare the oncological results and effects of conservative treatment in IPP with conservative treatment in EPP or TURBT without perforation. In this study, we aimed to determine the clinical course and oncological results of patients who underwent Foley catheter placement for bladder perforation, especially IPP.

\section{Materials and Methods}

\section{Patient Data Collection Using CDW}

We reviewed the surgical records of 4,543 patients who underwent 26-French monopolar TURBT for bladder cancer from January 2000 to December 2017. The following keywords were entered into the CDW system (SUPREME) of Seoul National University Hospital (SNUH) EHR to select the patients with the keyword in the surgical record. The records compiled by the 4 surgeons were analyzed. The keywords were perforation, perforated, fat, distension, extravasation, and leakage. The case output from the CDW was secondarily reviewed by a urologist. We defined bladder perforation as described in the EAU guideline: "visualization of fatty tissue, a dark space between detrusor muscle fibers, signs of major perforation such as the inability to distend the bladder, a low return of irrigation fluid, and abdominal distension, and visualization of the bowel" [12]. Patients with perforations mentioned in the surgical records were divided into the IPP and EPP groups. Patients whose perivesical fat was exposed during surgery but had no signs of major perforation were classified under the EPP group. We classified IPP as cases of direct bowel visualization through cystoscopy or accompanying signs of major perforation during TURBT. We analyzed the management procedures for perforations, pathologic results, treatment duration, complications, recurrence, and progression. We excluded patients with confirmed bowel injury with perforation during TURBT, T3, or no pathologic tumor diagnosis. Existing TURBT cohort group data of our hospital were used to design the control TURBT group without perforation.

\section{Clinical Evaluation}

The duration of Foley maintenance and cystography was determined by the surgeon according to the severity of intraoperative perforation. The presence of postoperative symptoms was evaluated to determine the need for surgical correction. Subsequently, patients were regularly followed up based on pathological tumor stage, grade, and risk evaluation. Also, the patients underwent cystoscopy, urinalysis, urine culture, complete blood count, chest radiography, and CT. Tumor recurrence and progression were confirmed by histological or imaging studies. Moreover, intra-abdominal metastasis, such as abdominal lymph node metastasis or mass recurrence, was recorded separately. We compared patient characteristics and clinicopathologic features in the IPP and EPP groups. In addition, the potential risk factors of open surgery were analyzed by comparing the IPP treated with Foley alone group and the IPP treated with the open surgery group. Recurrence-free survival (RFS) and progression-free survival (PFS) were compared in the IPP treated with Foley alone group and propensity-matched 
Table 1. Patients' characteristics and clinicopathologic features of the 2 perforation groups

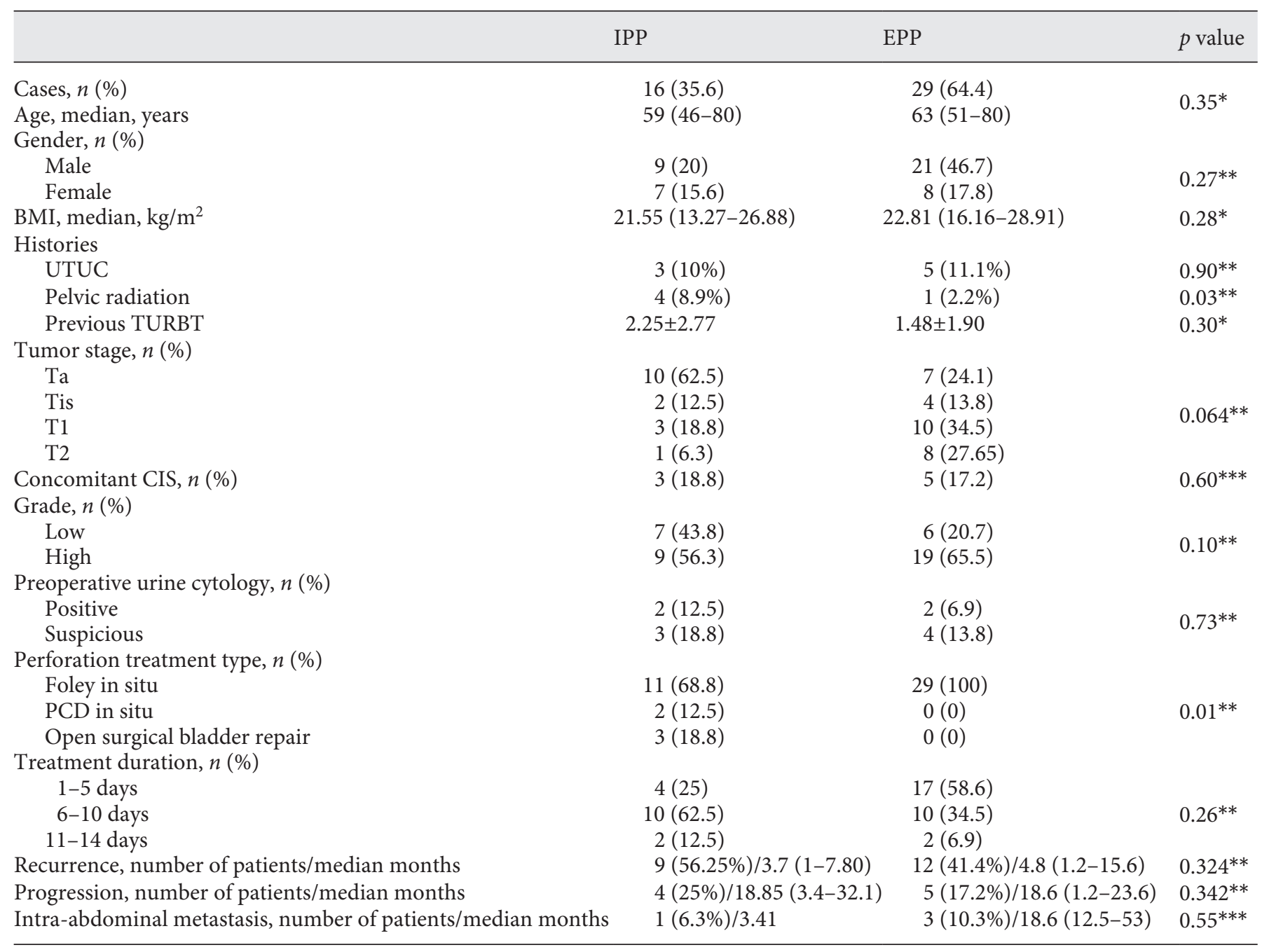

IPP, intraperitoneal perforation; EPP, extraperitoneal perforation; UTUC, upper tract urothelial cancer; TURBT, transurethral resection of bladder tumor; CIS, carcinoma in situ; PCD, percutaneous drainage. ${ }^{*}$ Mann-Whitney test. ${ }^{* *} \chi^{2}$ test. ${ }^{* * *}$ Fisher's exact test.

control TURBT group. Furthermore, we compared the RFS and PFS in the IPP treated with Foley alone group and the EPP group to evaluate the prognosis of different perforation types treated with Foley drainage alone.

\section{Statistical Analysis}

Continuous variables were expressed as mean \pm standard deviation or median. We used the Mann-Whitney $U$ test, the $\chi^{2}$ test, or Fisher's exact test to analyze the differences in the clinicopathologic parameters. Kaplan-Meier survival curves were used to determine RFS and PFS, and $p<0.05$ was considered statistically significant. Through the matching process, 1:5 propensity score matching was used to match 55 patients in the control TURBT group with the patients in the IPP Foley only group and to compare RFS with PFS. The covariates for propensity score matching were T stage, presence of CIS, grade, tumor size, number of tumors, age, sex, and BMI.

\section{Results}

\section{Clinicopathologic Characteristics and the Course of Patients with Perforation}

During the TURBT procedure, 45 patients $(0.99 \%)$ had bladder perforations. Of the 45 patients, $29(64.4 \%)$ and 16 (35.6\%) had EPP and IPP, respectively. The 2 groups showed differences in the history of previous pelvic radiotherapy (Table 1). All patients who had EPP were treated with Foley catheter drainage alone for a mean duration of 5.7 days without any complications. Sixteen IPP occurred in the bladder dome $\times 7$, posterior wall $\times 4$, anterior wall $\times 2$, left lateral wall $\times 2$, and right lateral wall $\times 1$. Of the 16 IPP, 2 patients had perforations larger than the 
Table 2. Comparison between the patient groups for the analysis of potential risk factors requiring open surgery in case of IPP

\begin{tabular}{|c|c|c|c|c|c|c|}
\hline IPP treated with open surgery $(n=3)$ & $65.67 \pm 13.80$ & $22.93 \pm 3.44$ & $6 \pm 1.73$ & $3 \pm 1.73$ & $3.33 \pm 3.21$ & $3(100 \%)$ \\
\hline$p$ value & $0.585^{*}$ & $0.585^{*}$ & $0.305^{*}$ & $0.346^{*}$ & $0.262^{*}$ & $0.011^{* *}$ \\
\hline
\end{tabular}

IPP, intraperitoneal perforation; TURBT, transurethral resection of bladder tumor. ${ }^{*}$ Mann-Whitney test. ${ }^{* *}$ Fisher's exact test.

Table 3. The characteristics of the patients with IPP and propensity-matched control TURBT; treatments of IPP with Foley alone

\begin{tabular}{lccc}
\hline & $\begin{array}{l}\text { IPP treated } \\
\text { with Foley } \\
(n=11)\end{array}$ & $\begin{array}{l}\text { Propensity-matched } \\
\text { control TURBT } \\
(n=55)\end{array}$ & $p$ value \\
\hline Age, mean, years & $59.64 \pm 10.24$ & $60.13 \pm 13.34$ & $0.711^{*}$ \\
Sex, $n(\%)$ & $7(63.6)$ & $29(52.7)$ & $0.742^{* * *}$ \\
$\quad$ Male & $4(36.4)$ & $26(47.3)$ & $0.897^{*}$ \\
Female & $21.90 \pm 3.26$ & $21.83 \pm 3.53$ & $0.795^{*}$ \\
BMI, mean, kg/m $\mathrm{m}^{2}$ & $18.09 \pm 14.78$ & $18.32 \pm 12.03$ & $0.711^{*}$ \\
Tumor size, mm & $6.09 \pm 5.39$ & $5.37 \pm 4.97$ & \\
Tumor number & 8 & 33 & $0.744^{* *}$ \\
T stage & 1 & 8 & $0.668^{* * *}$ \\
Ta & 1 & 11 & \\
Tis & 1 & 3 & $0.857^{* *}$ \\
T1 & 2 & 8 & \\
T2 & 4 & 21 & \\
Concomitant CIS & 6 & 26 & \\
Grade & & & \\
Low & & & \\
High & & & \\
\hline
\end{tabular}

IPP, intraperitoneal perforation; TURBT, transurethral resection of bladder tumor; CIS, carcinoma in situ. ${ }^{*}$ Mann-Whitney test. ${ }^{* *} \chi^{2}$ test. ${ }^{* * *}$ Fisher's exact test. size of the cystoscope, and the other patients had a smaller size than that of the cystoscope. Eleven patients with IPP were treated with the Foley catheter alone. Two patients had immediate postoperative PCD for 4 and 7 days. Three patients finally underwent open surgical bladder repair of perforations. Among the patients who underwent surgery, 2 patients who had nonresolution of the perforation after cystography on postoperative days (PODs) 6 and 11 without any symptoms underwent delayed open surgical bladder repair, and 1 patient with persistent abdominal distension and pain due to malfunctioning of Foley drainage underwent delayed open surgical bladder repair on POD 1. All patients who underwent surgery had a history of previous radiation therapy which was significantly different from the patients who showed improvement with Foley alone (Table 2). No delayed postoperative bowel perforation or sepsis was observed in the IPP group. Intra-abdominal recurrence during follow-up was not significantly different between the 2 perforation groups. Table 3 shows the patient characteristics of the IPP treated with Foley alone group and the matched control TURBT group.

\section{Recurrence and Progression after Treatments for Perforation}

We have presented the recurrence and progression data of the 2 perforation groups in Table 1 . In the IPP treated with the Foley alone group, bladder cancer recurrence occurred after a mean of 3.3 months in $5(45.5 \%)$ patients, and progression was seen in 1 patient $(9.1 \%)$ at 


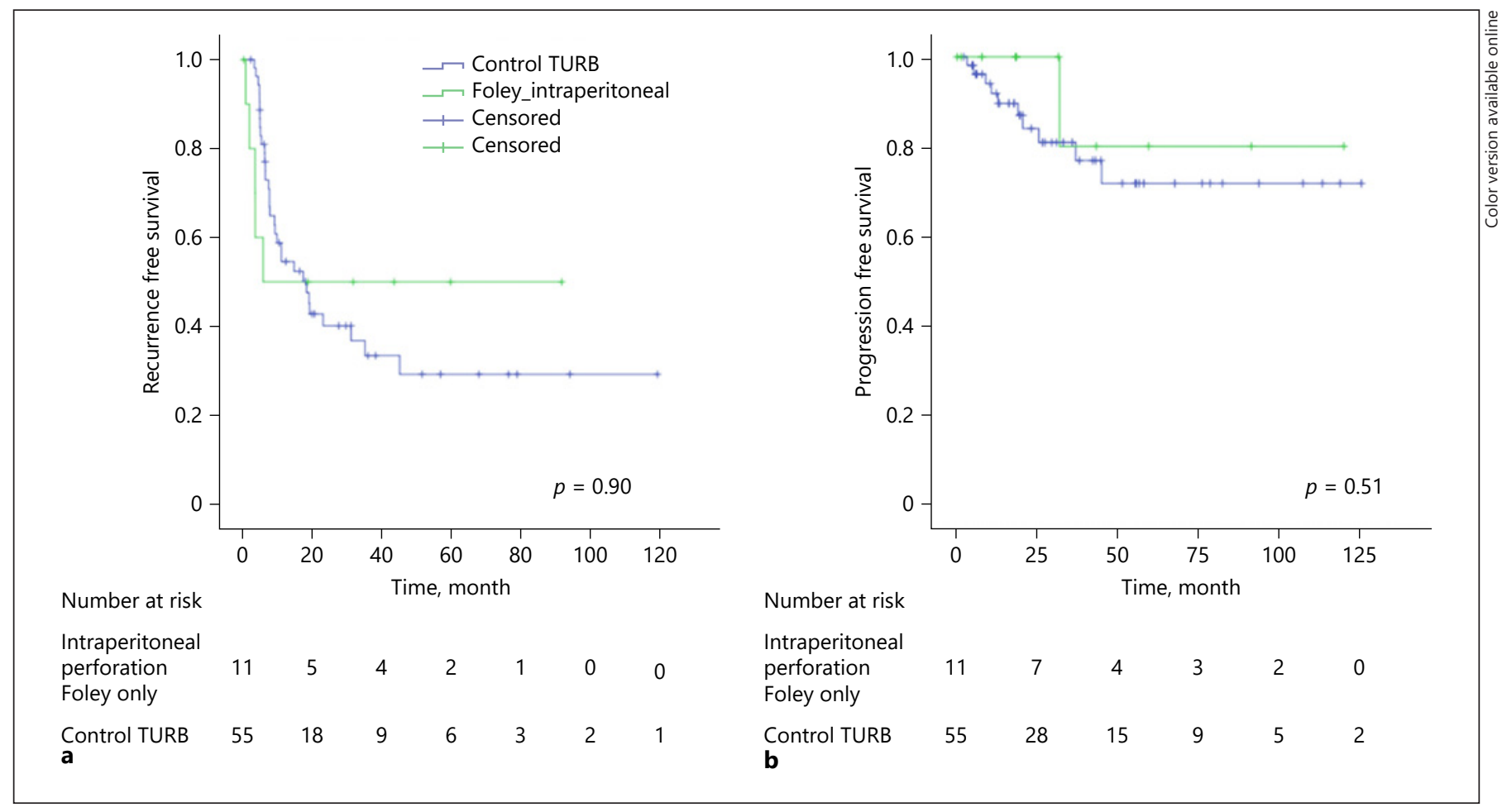

Fig. 1. Kaplan-Meier RFS (a) and PFS (b) in the Foley alone treated patients in the IPP group compared with the matched control TURBT group. The median follow-up duration was $31.8(0.4-120.3)$ months for the IPP treated with the Foley catheter alone group and 27.7 (2.5-129.1) months for the control TURBT group. RFS, recurrencefree survival; PFS, progression-free survival; IPP, intraperitoneal perforation; TURBT, transurethral resection of bladder tumor.

32.1 months after treatment. Figures 1 and 2 show RFS and PFS in the IPP treated with Foley alone group compared with those in the other groups. RFS and PFS exhibited no significant difference between the IPP treated with Foley alone group and the control TURBT group ( $p=0.90, p=0.51)$ and between the IPP treated with Foley alone group and the EPP group $(p=0.45, p=0.69)$.

\section{Discussion}

\section{Perforation Occurrence in Prior Studies}

Complications of TURBT often occur during deep and wide excisions that are generally performed for complete resection [13-15]. Usually, the perforation occurrence rate is also low, ranging between 1.3 and 5\%. It is known that IPP accounts for $10-20 \%$ of total perforations; however, the reported perforation rate might be underestimated [1]. In a prospective study, El Hayek et al. [16] reported that 34 patients had no intraoperative perforations; nonetheless, 17 (50\%) were found to have extravesical-type contrast leakage on routine cystography. Apparently, it can be understood that we do not notice many cases of EPP occurrences, perhaps as it is not easy to find small perforations, and they may be self-limiting through the routine postoperative care process. In this study, $45(0.99 \%)$ of 4,543 patients were found to have perforations, and IPP accounted for $35.6 \%$ of total perforations. The reason that the probability of occurrence of EPP was slightly low could be because the operator's subjectivity was acted upon according to the characteristics of EPP and thus not recorded at all situations of occurrence. Due to the low incidence and underestimation, there are few studies on IPP, and no data have been reported on the outcome of conservative treatment with the Foley catheter.

\section{Using CDW for Data Collection}

Studies on perforation are few and have small sample sizes. It is difficult to study a clinical situation with a small sample population due to a low incidence over a long period of time. Using CDW, we were able to find 45 cases 


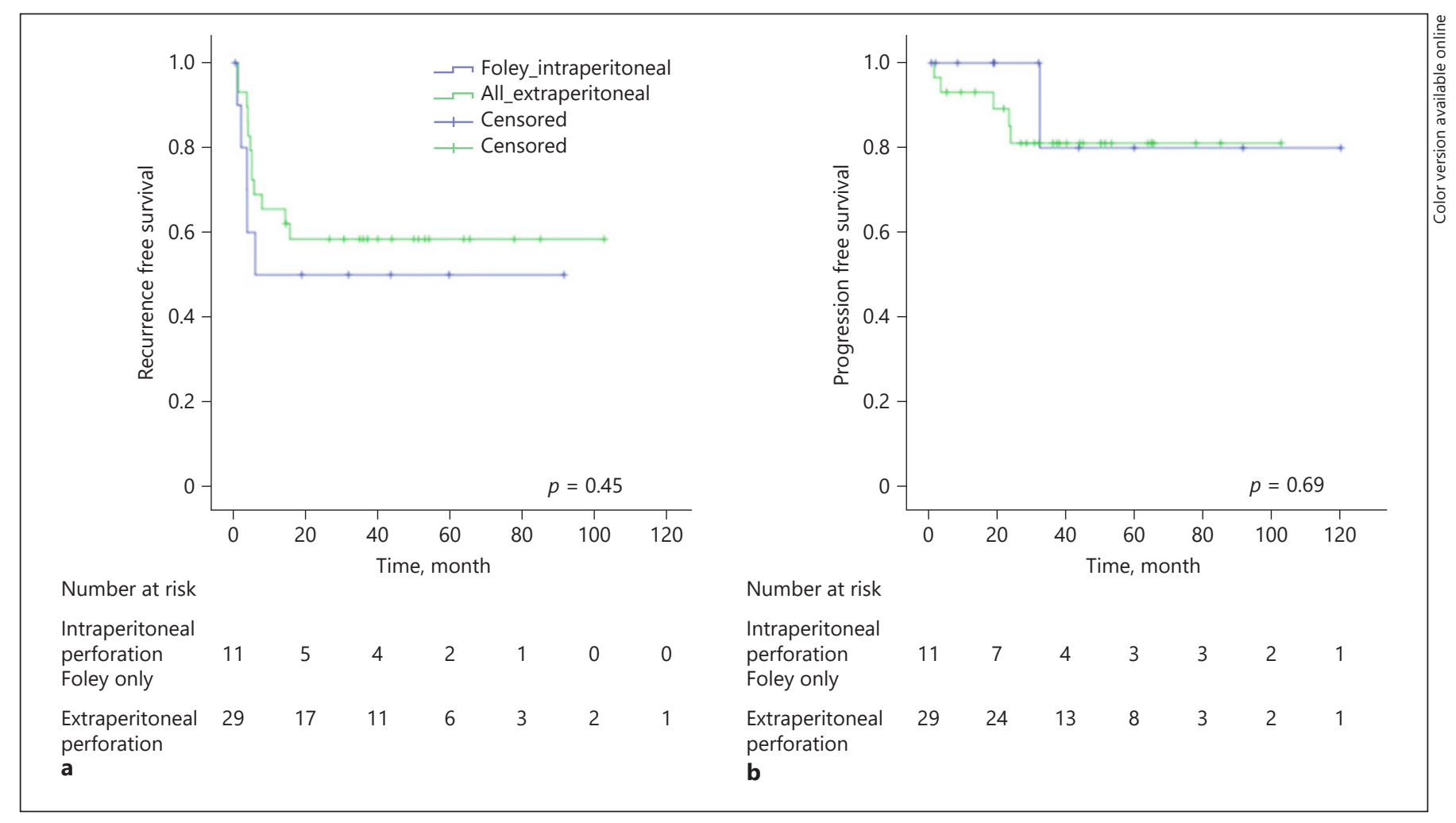

Fig. 2. Kaplan-Meier RFS (a) and PFS (b) in patients treated with the Foley catheter alone in the IPP group compared with those in the EPP group. The median follow-up duration was $31.8(0.4-120.3)$ months for the IPP treated with the Foley catheter alone group and 42.7 (4.9-136.8) months for the EPP group. RFS, recurrence-free survival; PFS, progression-free survival; IPP, intraperitoneal perforation; EPP, extraperitoneal perforation.

among the 4,543 surgical cases that were practically impossible to search. This was possible because of the ability to quickly output data by selecting patients with keywords in free-text records. Escudié et al. [17] reported the results of using CDW to identify autoimmune comorbidities among patients with celiac disease. Two physicians took only $2 \mathrm{~h}$ to find 15 autoimmune comorbidities from 6,340 documents with 741 patients. They used the keyword "celiac disease" from a text document (discharge or letter), prescriptions of insulin with levothyroxine, and the ICD-10 code as queries to find. As such, the CDW can be useful for obtaining essential information from massive clinical data [18].

\section{A Trend toward Conservative Management}

Armenakas et al. [19] reported good long-term followup findings ( $98.4 \%$ success rate of repair) of the surgical approach in iatrogenic bladder perforations and no delay in bladder cancer treatment with prompt intraoperative recognition. However, technological advancement is a reason for active conservative management. Bipolar tech- nology can deliver energy only to the focal area. Additionally, isotonic fluid has a low risk of complex absorption, and now PCD intervention is more advanced compared with the past [20]. These points are the basis for stepping to minimally invasive conservative treatment. If there is no evidence of bowel injury when IPP occurs, Foley drainage and PCD can be considered initially in patients with perforation-related symptoms as minimally invasive management, instead of surgical correction. Conservative management may be attempted when IPP occurs with small-sized perforations in the absence of peritonitis, ileus, or bowel injury; however, related data are limited in the literature $[5,12,21,22]$. Some studies wherein a small number of patients were treated with the Foley catheter and PCD placement reported safe and effective improvement $[5,12,21]$. In the field of gynecology, Alperin et al. [22] reported a case of improved Foley catheter drainage when delayed bladder IPP was diagnosed after hysterectomy. Therefore, even in IPP that occurs during TURBT, treatment with Foley alone can be carefully tried, and the results of this study may be the first 
step in securing evidence. Of the 14 patients who tried the Foley catheter, treatment was terminated in 11 patients without any sequelae, except in the case of 3 patients with a larger perforation than the cystoscope.

\section{Principal Findings}

In our study, 11 patients treated with a Foley catheter alone were discharged with uneventful recoveries. Two patients who underwent postoperative PCD had similar results. Three patients who were treated initially with the Foley catheter alone underwent delayed open bladder repair surgery on PODs 1, 6, and 11. Patients who underwent delayed surgery on PODs 6 and 11 without symptoms had persistent perforations on postoperative cystography. Therefore, $11(78.6 \%)$ of the 14 patients who were initially treated with the Foley catheter showed conservative improvement. Although 3 patients (21.4\%) underwent delayed open surgery, there were neither sequelae nor therapeutic delays. All 3 patients who underwent delayed surgery had previously been treated with pelvic radiotherapy due to colon cancer or cervical cancer. In this study, radiation therapy was analyzed as a potential risk because treatment with Foley alone would not be sufficient for IPP. Radiation therapy has been reported to cause defunctionalized bladder, cystitis, and even spontaneous bladder rupture by chronic fibrosis [23]. Several studies described that radiation therapy, pelvic surgery, and Foley indwelling can weaken the structure of the bladder wall by causing histologic changes including fibrosis, inflammation, and necrosis $[24,25]$. Of the 2 patients with the size of the perforation larger than the cystoscope and treated with the Foley catheter alone, only 1 showed improvement with conservative treatment and the other had delayed surgery on POD 1 due to malfunction of the Foley drainage. The number of cases was small; however, in cases of perforations larger than the cystoscope and history of pelvic radiotherapy, it may be safe to perform open surgical bladder repair.

In patients with high-risk bladder cancer, adjuvant Bacillus Calmette-Guérin (BCG) instillation should be considered actively because of the advantages in overall survival and cancer-specific mortality [26]. However, it is known that tuberculosis-induced peritonitis is very rare, occurring 2-3 years after BCG instillation, and raises the suspicion of immunodeficiency or a history of continuous ambulatory peritoneal dialysis [27]. In 1 patient with IPP, tuberculosis-induced peritonitis occurred 5 months after BCG induction when the Foley catheter was removed with cystography after 1 week. It is difficult to find a clear association between the abovementioned factors; however, it seems necessary to perform cystography to confirm the absence of extravasation [28].

Bladder perforation during TURBT can increase the risk of recurrence and progression of bladder cancer. Pycha and colleagues [29] noted that patients with bladder perforation including IPP and EPP during TURBT had shorter disease-free survival and $\mathrm{T}$ stage progression of the recurrent disease of bladder cancer. In this study, the incidence of extravesical recurrence varies from 0 to $13.3 \%$ $[1,4,6]$. Intra-abdominal recurrence was observed in 1 $(6.3 \%)$ and $3(10.3 \%)$ patients with IPP and EPP, respectively. In our study, intraperitoneal metastasis was observed 3.4 months later in a patient of the IPP group. This patient had a perforation larger $(1.5 \mathrm{~cm})$ than the size of cystoscopy and had open bladder repair on POD 1 due to the malfunctioning of Foley drainage. The TURBT result of this patient was T1 high grade. Interestingly, Skolarikos et al. [4] mentioned open surgical repair following IPP and tumor size as risk factors for extravesical recurrence. All patients with extravesical tumor recurrences or evidence of metastatic disease had previously undergone open surgical repair [30,31]. The authors stated that this might be due to an increased amount of tumor cell spillage during open surgery. In the conservative management of this study, the RFS and PFS oncological outcomes of the IPP treated with Foley alone group were compared with those of the control TURBT and the EPP groups; nonetheless, there were no significant differences (Fig. 1,2). Therefore, conservative management can be tried considering the risk factors of pelvic radiation history and size when IPP occurs. As there exist no studies on the comparison between RFS and PFS of the IPP treated with Foley alone with the EPP or control TURB, the results of this study may help to determine conservative treatment for IPP.

\section{Limitations}

Although we retrospectively analyzed many patients, the number of patients with perforations was small. Consequently, the standard treatment for perforations and factors necessary for decision-making are not established. The absence of a standard recording form for perforation evaluation and the dependence on the operator's subjective records also led to the lack of specific information on perforations. Thus, the record of the location of the perforations or size was insufficient. Also, EPP was often not noted on the surgical record as the occurrence was relatively frequent and likely to improve without any complications. There was also a variation in the management of some cases because of the absence of a standard consensus on tests, postoperative management, and postperforation follow-up. For ex- 
ample, not all patients underwent cystography at the end of every TURBT, and small persistent perforations were likely missed. For these reasons, the inconsistent clinical management of patients with IPP might have been a study limitation. As reported, even in big hospitals, IPP occurs very rarely. However, clinically, urologists are interested in the treatment of IPP and the prognosis of minimally invasive treatment. Thus, while this small number of cases may not be sufficient to draw solid conclusions, urologists depend on these findings in the appropriate IPP treatment. Additionally, multicenter large-scale studies are needed to provide findings with a high level of evidence.

\section{Conclusion}

The incidence of intraperitoneal bladder perforation during TURBT was low, which was consistent with the findings of the previous studies. In the case of IPP of a size smaller than the cystoscope and without previous pelvic radiotherapy history, patients treated with Foley catheter drainage alone showed improvement without complications or therapeutic delays. Furthermore, the RFS and PFS oncological outcomes were not significantly different from those in the control TURBT and EPP groups. Therefore, when an IPP of a size smaller than that of the cystoscope occurs and without previous pelvic radiotherapy history, Foley catheter drainage alone can be considered carefully as an initial treatment.

\section{Statement of Ethics}

This study was approved by the Institutional Review Board of Seoul National University College of Medicine/SNUH (SNUCM/ SNUH IRB) (No. H-1807-093-960). Owing to the retrospective nature of this study, the SNUCM/SNUH IRB waived the requirement to obtain informed consent from the patients. The study was conducted in compliance with the principles of the Declaration of Helsinki.

\section{Conflict of Interest Statement}

The authors have no conflicts of interest to declare.

\section{Funding Sources}

This work was supported by a Basic Science Research Program through the National Research Foundation of Korea (NRF) funded by the Ministry of Education (NRF-2019R1F1A1050507).

\section{Author Contributions}

J.L. and J.H.K. conceptualized this study. C.W.J., C.K., H.H.K., and J.H.K. performed and were responsible for the treatment of patients. J.L. and J.S. collected the patients' data and analyzed statistically. J.L. and J.H.K. drafted the manuscript. C.W.J., C.K., H.H.K., and J.H.K. validated the analysis and reviewed the manuscript. J.H.K. supervised this study and revised the final manuscript. All authors approved the results of the data analysis and the final manuscript.

\section{References}

1 Collado A, Chéchile GE, Salvador J, Vicente J. Early complications of endoscopic treatment for superficial bladder tumors. J Urol. 2000 Nov;164(5):1529-32.

2 Nieder AM, Meinbach DS, Kim SS, Soloway MS. Transurethral bladder tumor resection: intraoperative and postoperative complications in a residency setting. J Urol. 2005 Dec; 174(6):2307-9.

3 Morales A, Eidinger D, Bruce AW. Intracavitary Bacillus Calmette-Guerin in the treatment of superficial bladder tumors. J Urol. 1976 Aug;116(2):180-3.

4 Skolarikos A, Chrisofos M, Ferakis N, Papatsoris A, Dellis A, Deliveliotis C. Does the management of bladder perforation during transurethral resection of superficial bladder tumors predispose to extravesical tumor recurrence? J Urol. 2005 Jun;173(6):190811.

5 Gomez RG, Ceballos L, Coburn M, Corriere JN Jr, Dixon CM, Lobel B, et al. Consensus statement on bladder injuries. BJU Int. 2004 Jul;94(1):27-32.
6 Golan S, Baniel J, Lask D, Livne PM, Yossepowitch O. Transurethral resection of bladder tumour complicated by perforation requiring open surgical repair - clinical characteristics and oncological outcomes. BJU Int. 2011 Apr; 107(7):1065-8.

7 Pansadoro A, Franco G, Laurenti C, Pansadoro V. Conservative treatment of intraperitoneal bladder perforation during transurethral resection of bladder tumor. Urology. 2002 Oct;60(4):682-4.

8 Inmon WH, Imhoff C, Sousa R. Corporate information factory. 2nd ed. New York: John Wiley \& Sons; 2001.

9 Schubart JR, Einbinder JS. Evaluation of a data warehouse in an academic health sciences center. Int J Med Inform. 2000 Dec;60(3):319-33.

10 Perry T. Disease management. Technologydriven outcomes. Health Manag Technol. 2004 Jan;25(1):40-3.

11 Karami M, Rahimi A, Shahmirzadi AH. Clinical data warehouse: an effective tool to create intelligence in disease management. Health Care Manag. 2017 Oct/Dec;36(4):380-4.
12 Summerton DJ, Kitrey ND, Lumen N, Serafetinidis E, Djakovic N; European Association of Urology. EAU guidelines on iatrogenic trauma. Eur Urol. 2012 Oct;62(4): 628-39.

13 Chang SS, Boorjian SA, Chou R, Clark PE, Daneshmand S, Konety BR, et al. Diagnosis and treatment of non-muscle invasive bladder cancer: AUA/SUO guideline. J Urol. 2016 Oct;196(4):1021-9.

14 Flaig TW, Spiess PE, Agarwal N, Bangs R, Boorjian SA, Buyyounouski MK, et al. NCCN guidelines insights: bladder cancer, version 5.2018. J Natl Compr Canc Netw. 2018 Sep; 16(9):1041-53.

15 Babjuk M, Burger M, Compérat E, Gontero P Mostafid AH, Palou J, et al. EAU guidelines on non-muscle-invasive bladder cancer. Presented at the EAU Annual Congress Barcelona; Arnhem, The Netherlands; 2019. Available from:
Efficacy of Foley Treatment of Intraperitoneal Bladder Perforation
Urol Int 2022;106:138-146

DOI: $10.1159 / 000517332$ 
16 El Hayek OR, Coelho RF, Dall'oglio MF, Murta CB, Ribeiro Filho LA, Nunes RL, et al. Evaluation of the incidence of bladder perforation after transurethral bladder tumor resection in a residency setting. J Endourol. 2009 Jul; 23(7):1183-6.

17 Escudie JB, Jannot AS, Zapletal E, Cohen S, Malamut G, Burgun A, et al. Reviewing 741 patients records in two hours with FASTVISU. AMIA Annu Symp Proc. 2015;2015:5539.

18 Mohammed RO, Talab SA. Clinical data warehouse issues and challenges. IJUNESST. 2014;7(5):251-62.

19 Armenakas NA, Pareek G, Fracchia JA. Iatrogenic bladder perforations: longterm followup of 65 patients. J Am Coll Surg. 2004 Jan; 198(1):78-82.

20 Wang DS, Bird VG, Leonard VY, Plumb SJ, Konety B, Williams RD, et al. Use of bipolar energy for transurethral resection of bladder tumors: pathologic considerations. J Endourol. 2004 Aug;18(6):578-82.
21 Traxer O, Pasqui F, Gattegno B, Pearle MS. Technique and complications of transurethral surgery for bladder tumours. BJU Int. 2004 Sep;94(4):492-6.

22 Alperin M, Mantia-Smaldone G, Sagan ER. Conservative management of postoperatively diagnosed cystotomy. Urology. 2009 May; 73(5):1163-9.

23 Elliott SP, Malaeb BS. Long-term urinary adverse effects of pelvic radiotherapy. World J Urol. 2011 Feb;29(1):35-41.

24 Haddad FS, Wachtel TL. Spontaneous intraperitoneal rupture of the bladder. Urol Int. 1987;42(6):467-9.

25 Meyer K, Welsch H. [Spontaneous rupture of the urinary bladder after radiotherapy]. Zentralbl Chir. 1993;118(4):230-1.

26 Novotny V, Froehner M, Ollig J, Koch R, Zastrow S, Wirth MP. Impact of adjuvant intravesical Bacillus Calmette-Guérin treatment on patients with high-grade T1 bladder cancer. Urol Int. 2016;96(2):136-41.
27 Soylu A, Ince AT, Polat H, Yasar N, Ciltas A, Ozkara S, et al. Peritoneal tuberculosis and granulomatous hepatitis secondary to treatment of bladder cancer with Bacillus Calmette-Guérin. Ann Clin Microbiol Antimicrob. 2009 Apr 15;8:12.

28 Balbay MD, Cimentepe E, Unsal A, Bayrak O, Koç A, Akbulut Z. The actual incidence of bladder perforation following transurethral bladder surgery. J Urol. 2005 Dec;174(6): 2260-3; discussion 62-3.

29 Comploj E, Dechet CB, Mian M, Trenti E, Palermo S, Lodde M, et al. Perforation during TUR of bladder tumours influences the natural history of superficial bladder cancer. World J Urol. 2014 Oct;32(5):1219-23.

30 Mydlo JH, Weinstein R, Shah S, Solliday M, Macchia RJ. Long-term consequences from bladder perforation and/or violation in the presence of transitional cell carcinoma: results of a small series and a review of the literature. J Urol. 1999 Apr;161(4):1128-32.

31 Chakravarti A, Day DW, MacDermott S. Extravesical transitional cell carcinoma as a result of implantation after perforation of the bladder. BJU Int. 2000 Jun;85(9):1150-1. 